\title{
Josephson Junctions Like Behavior in Superconducting Nb Films with Diluted Triangular Arrays of Antidots
}

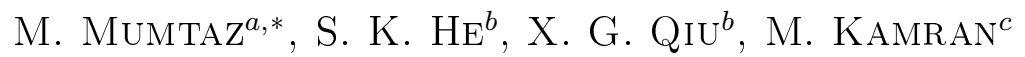 \\ ${ }^{a}$ Materials Research Laboratory, Department of Physics FBAS, International Islamic University (IIU), \\ Islamabad 44000, Pakistan \\ ${ }^{b}$ Beijing National Laboratory for Condensed Matter Physics, Institute of Physics, \\ Chinese Academy of Science, Beijing 100190, China
}

${ }^{c}$ Department of Electrical Engineering, COMSATS Institute of Information Technology, Wah Cantt, Pakistan

\begin{abstract}
We present current versus voltage $(I-V)$ measurement on superconducting $\mathrm{Nb}$ films with diluted triangular arrays (honeycomb and kagome) of antidots. Peaks and dips appearing in the derivative $(d V / d I)$ of $I-V$ curves showing the variation in the resistance is the indication of Josephson Junctions like weak-links in these systems. These observations also reveal the presence of mobile interstitial vortices in these lattices with regular pinning arrays. This behavior is predominant in honeycomb arrays due to large density of interstitial spaces in this system as compared that of to kagome lattice. This behavior can be tuned by temperature as well as external magnetic field but the tuning values of these parameters can be different for different symmetries of antidots.
\end{abstract}

DOI: $10.12693 /$ APhysPolA.127.898

PACS: 74.25.F-, 74.78.-w, 74.81.Fa

\section{Introduction}

Appreciable efforts have been exercised for the improvements of critical current density $\left(J_{C}\right)$ in superconductors through different techniques. There are many reasons for dissipative mechanism in superconducting materials but the main reason is the unwanted motion of vortices. Pinning of the vortices is one of the most commonly employed mechanisms used to increase and fix the $J_{c}$ of the superconductors. Many attempts were made to enhance the pinning of vortices in high $T_{c}$ superconductors by creating structural defects using various samples treatments, especially by energetic irradiation processes. Also by using lithography, it has become possible to create well-defined periodic nanostructured arrays for periodic pinning centers with definite size, depth, periodicity, and density. This method has been employed for the enhancement of $J_{c}$ in high $T_{c}$ as well as conventional superconductors [1-5]. Investigations of hole arrays are needed to better understand the physics related to superconductors with micro/nano structures. A number of intersecting and novel phenomena like commensurate effects [6], reconfiguration [7] and rectification effects [8] etc, are explored in superconducting films with artificial pinning arrays of well-defined size, shape and composition. In this work, we studied the transport properties of superconducting $\mathrm{Nb}$ films with honeycomb and kagome arrays of antidots. Here we focused especially on $I-V$ characteristics of the samples with two dimensional periodic potential created by antidots. In spite of very intensive

${ }^{*}$ corresponding author; e-mail: mmumtaz75@yahoo.com theoretical research, a limited number of experimental investigations have been carried out on these systems. Due to small coherence length of $\mathrm{Nb}$ and $\mathrm{NbN}$ materials, the pinning centers trap mutiquanta vortices whereas a single vortex occupation was assumed theoretically, therefore, one-to-one correspondence between the experiment and theory could not be satisfied. This weakly pinned interstitial lattice can be easily moved through the potential created by the strongly pinned vortices at the antidots. When all the interstitial spaces are filled, it is to be expected that the repulsive interactions will lead to a coherent motion of all the interstitial vortices.

\section{Experimental details}

The nanostructured samples with honeycomb and kagome arrays of antidots on superconducting $\mathrm{Nb}$ films were prepared and the detailed procedure of preparation of these samples was already reported [9]. The superconducting $\mathrm{Nb}$ film with a thickness of $100 \mathrm{~nm}$ was deposited by magnetron sputtering on $\mathrm{Si}$ substrate with $\mathrm{SiO}_{2}$ buffer layer. A micro-bridge for four terminal transport measurements was fabricated by ultraviolet photolithography followed by reactive ion etching. Then the desired arrays covering the whole bridge area of $60 \times 60 \mu \mathrm{m}^{2}$ was patterned by electron beam lithography on a polymethyl metacrylate (PMMA) resist layer. Finally, the pattern was transferred to the $\mathrm{Nb}$ film by magnetically enhanced reactive ion etching.

The $I V$-measurements were carried out at different temperatures just below the zero resistivity critical temperature $\left\{T_{c}(0)\right\}$ with and without external applied magnetic field by a commercial Physical Properties Measurement System (PPMS) manufactured by Quantum Design. The temperature stability was kept at $2 \mathrm{mK}$ during these measurements. 


\section{Results and discussion}

Resistance versus temperature measurements of honeycomb and kagome samples are shown in Fig. 1 . The zero resistivity critical temperatures $\left\{T_{c}(0)\right\}$ are $8.57 \mathrm{~K}$ for the honeycomb sample and $8.4 \mathrm{~K}$ for the kagome sample, respectively at probing current of $10 \mu \mathrm{A}$ in zero applied field. The value of $T_{c}(0)$ for honeycomb patterned sample is slightly greater than that of kagome arrays sample, which may be due to larger number of interstitial spaces in honeycomb as compared to that in kagom e lattice. The average diameter of the interstitial regions at temperatures $8.50 \mathrm{~K}$, approximately $520 \mathrm{~nm}$, is about six times larger than the coherence length. At even lower field such as $H / H 1=2,3$ in these systems, it is expected that the vortices can sit in each interstitial hexagonal plaquette. The strong inter-plaquette vortexvortex interaction favor the channeling of the vortices but the external field diminishes this channeling effect. The scanning electron micrograph (SEM) images of (a) honeycomb and (b) kagome arrays are shown in the inset of Fig. 1. The average diameter of the antidots in both the honeycomb and kagome arrays samples is about $340 \mathrm{~nm}$ and the distance between the two nearest neighbor holes is $400 \mathrm{~nm}$. It can be seen very clearly that $T_{c}(0)$ shifts to low temperature and superconducting transition becomes broadened in the kagome sample as compared to honeycomb sample, which is due to small interstitials regions and may be due to frustration phenomenon occurring in kagome lattice. Also the suppression of $T_{c}(0)$ in both the systems as compared to the unpatterned Nb superconducting thin film (i.e. $T_{c}(0)=8.66 \mathrm{~K}$ ) may be due to lithographic processing during preparation of these samples.

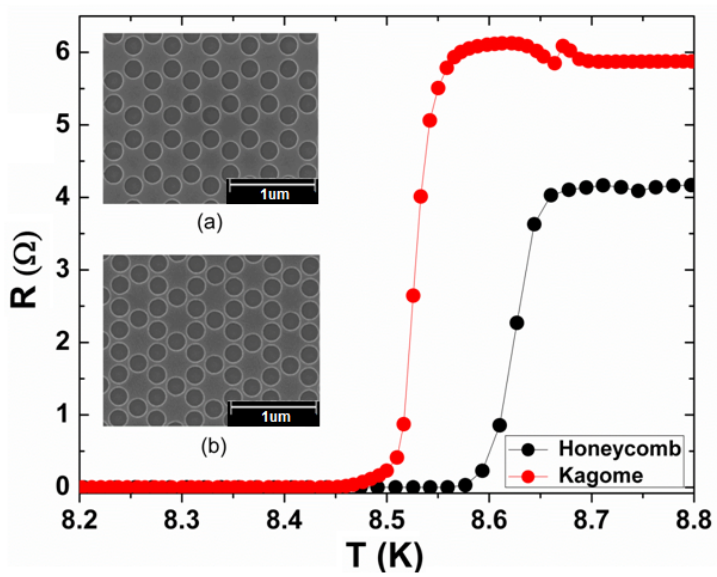

Fig. 1. The resistance versus temperature curves for honeycomb and kagome samples. The inset shows the SEM images (a) honeycomb and (b) kagome arrays array of holes.

The current versus voltage $(I-V)$ and $d V / d I$ versus $I$ measurements at different temperatures from 8.2 to $8.6 \mathrm{~K}$ and in zero field $(H=0)$ for honeycomb arrays sample are shown in Fig. 2. The giant Shapiro [10] like steps are also observed at almost all temperatures. The derivatives $(d V / d I)$ of $I-V$ curves show the variation in differential resistance in the form of spikes shown in (b) of Fig. 2. This junction like behavior has become prominent at temperature $8 . \mathrm{K}$ and $8.5 \mathrm{~K}$ just below $T_{c}(0)$. At very low and above $T_{c}(0)$, the junction behavior almost vanishes. At high temperature above $T_{c}(0)$ due to thermal fluctuations, the junction like behavior become diminished.

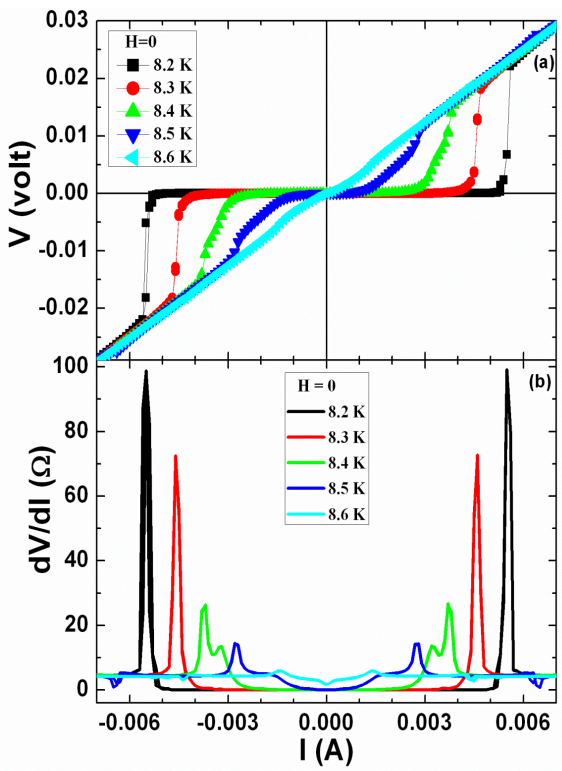

Fig. 2. (a)The current versus voltage $(I-V)$ characteristics of honeycomb arrays sample at different temperatures from 8.2 to $8.6 \mathrm{~K}$ in zero field $(H=0)$. (b) Differential resistance extracted from $I-V$ curves for honeycomb arrays sample at different temperatures from 8.2 to $8.6 \mathrm{~K}$ in zero field $(H=0)$.

At very low temperature the junctions start to overlap and the sample behave like normal unpatterned film. This is very useful information from application view point that the junction-like behavior can be tuned with the temperature. This behavior also depends upon the different parameters like hole size, patterning of the holes, etc.

$I-V$ and $d V / d I$ versus $I$ measurements at different temperatures from 8.2 to $8.6 \mathrm{~K}$ and in zero field $(H=0)$ for kagome lattice are shown in Fig. 3. The critical current $\left(I_{c}\right)$ for kagome lattice is less than that for honeycomb arrays sample.

Also the junction like behavior is very obvious at 8.2 , 8.3 , and $8.4 \mathrm{~K}$ from $d V / d I$ versus $I$ measurements as shown in (b) of Fig. 3. This is due to the characteristic structure of kagome lattice. The different heights of the peaks in $d V / d I$ versus $I$ in both the honeycomb arrays as well as in kagome lattice determine that the junctions are not absolutely identical. It is not practical to characterize the individual junction by probing every center between 


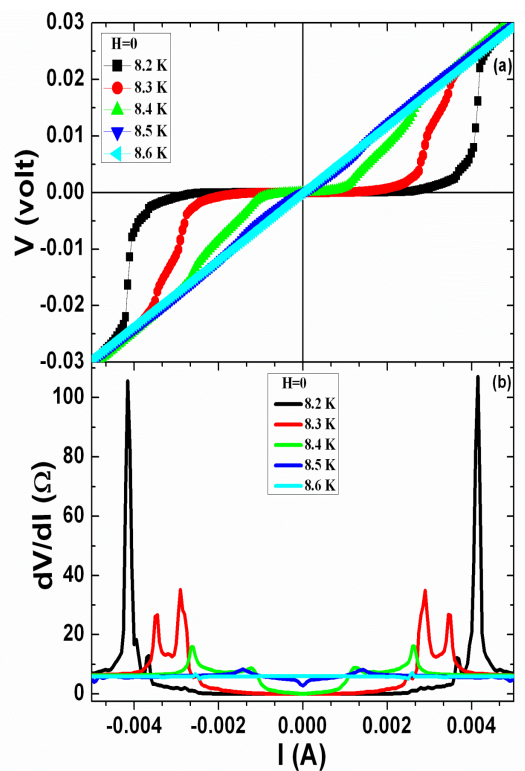

Fig. 3. (a) The current versus voltage $(I-V)$ characteristics of kagome patterned sample at different temperatures from 8.2 to $8.6 \mathrm{~K}$ in zero field $(H=0)$. (b) Differential resistance extracted from $I-V$ curves for kagome patterned sample at different temperatures from 8.2 to $8.6 \mathrm{~K}$ in zero field $(H=0)$.

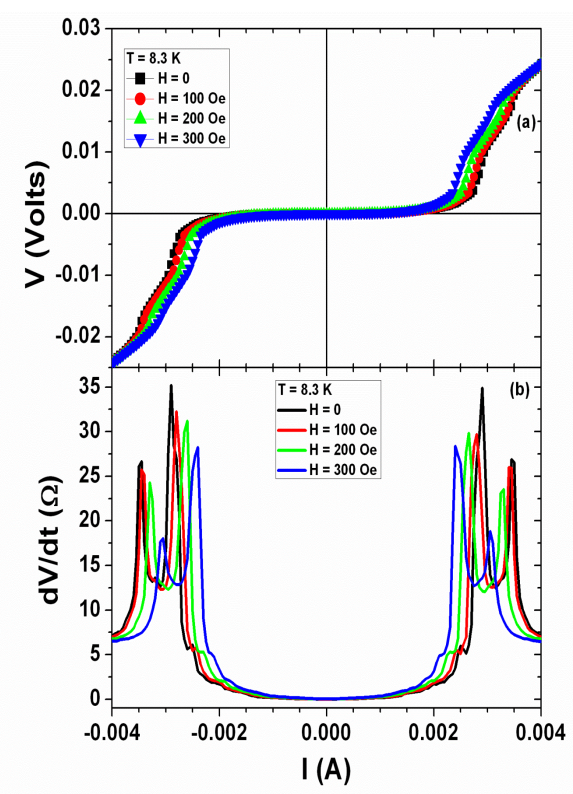

Fig. 4. (a) The current versus voltage $(I-V)$ characteristics of kagome patterned sample at different fields from 0 to 300 Oe at fixed temperature $T=8.3 \mathrm{~K}$. (b) Differential resistance extracted from $I-V$ curves for kagom'e patterned sample at different fields from 0 to 300 Oe at fixed temperature $T=8.3 \mathrm{~K}$. the adjacent junctions for large number of arrays. $I-V$ and $d V / d I$ versus $I$ measurements at $8.3 \mathrm{~K}$ and in different fields from 0 to 300 Oe for kagome lattice are also shown in Fig. 4.

It can be observed that external magnetic field can tune this behavior very beautifully. These experiments also reveal that there is a very weak suppression of critical current with the application of fields, which is a very good sign for applications.

\section{Conclusion}

We have studied the transport properties especially $I-V$ measurements at different temperatures and fields for superconducting $\mathrm{Nb}$ thin films with honeycomb and kagom'e arrays of antidots of very small edge-to-edge separation. The critical currents in kagom'e lattice are smaller than those measured in honeycomb arrays sample. We observe the dips and peaks in the derivative $(d V / d I)$ of $I V$-curves showing the abrupt variation in the resistance, which also indicates the Josephson Junctions like weak-links in these systems. These observations also reveal the mobility of interstitial vortices with regular pinning arrays. This behavior can be tuned by temperature as well as external applied magnetic field for application purpose but the tuning values of these parameters can be different for different symmetries of antidots.

\section{Acknowledgments}

Authors would like to thank Higher Education Commission (HEC) of Pakistan for financial support.

\section{References}

[1] C. Reichhardt, C.J. Olson, F. Nori, Phys. Rev. B 58, 6534 (1998).

[2] M. F. Laguna, C. A. Balseiro, D. Dominguez, F. Nori, Phys. Rev. B 64, 104505 (2001).

[3] C. Reichhardt, G.T. Zimányi, N. Gronbech-Jensen, Phys. Rev. B 64, 014501 (2001).

[4] C.C.de Souza Silva, J.V. de Vondel, B.Y. Zhu, M. Morelle, V.V. Moshchalkov, Phys. Rev. B 73, 014507 (2006).

[5] R. Cao, T.C. Wu, P.C. Kang, J.C. Wu, T.J. Yang, L. Horng, Solid Stat. Commun. 143, 171 (2007).

[6] M. Baert, V.V. Metlushko, R. Jonckheere, V.V. Moshchalkov, Y. Bruynseraede, Phys. Rev. Lett. 74, 3269 (1995)

[7] J.I. Martín, M. Vélez, A. Hoffmann, I.K. Schuller, J.L. Vicent, Phys. Rev. Lett. 83, 1022 (1999).

[8] J. E. Villegas, S. Savelév, F. Nori, E.M. Gonzalez, J.V. Anguita, R. Garcia, J.L. Vicent, Science 302, 1188 (2003).

[9] S.K. He, W.J. Zhang, H.F. Liu, G.M. Xue, B.H. Li, H. Xiao, Z.C. Wen, X.F. Han, S.P. Zhao, C.Z. Gu, X.G. Qiu, J. Phys.: Condens. Matter 24, 155702 (2012).

[10] S. Shapiro, Phys. Rev. Lett. 11, 80 (1963). 